\title{
El aprendizaje de la construcción arquitectónica a partir de los contenidos procedimentales y actitudinales
}

\author{
JORGE ROA FERNÁNDEZ \\ Universidad de Sevilla \\ Departamento de Construcciones \\ Arquitectónicas I \\ jroa@us.es \\ D.0.I.: http://dx.doi.org/10.12795/JDU.2018.i01.35 \\ Pp.: 619-636
}

\section{Resumen}

La singularidad de la docencia de la construcción en los últimos cursos de Arquitectura reside en que se manejan conceptos que ya vienen estudiados de cursos anteriores, y en estas últimas asignaturas se le impregna de un carácter más técnico y sobre todo direccionado a la práctica profesional del arquitecto. Ésta se subdivide en dos ámbitos muy diferenciados y estimulantes. Es en el estudio donde se proyecta y diseña lo que se va a construir, para posteriormente, en obra, llevar a cabo ese proceso constructivo con el arquitecto como director, y el proyecto como guía. La experiencia de innovación docente realizada en Construcción 6, de 5o curso en la ETSA de Sevilla tuvo como objetivo motivar a los estudiantes y poner en sus manos una metodología de análisis y toma de decisiones para aplicarla en la dirección de la obra. 
Palabras clave: Construcción 6, Grado en Arquitectura, Docencia Universitaria, Innovación Docente Universitaria, Dirección de Obra.

\section{Descripción del contexto}

La asignatura de Construcción 6 es del último curso de la carrera de Grado de Arquitectura (50). Es una asignatura básicamente práctica con un objetivo fundamental: aprender a desarrollar la actividad de Dirección de Obras por parte del Arquitecto y aprender a enfrentarse a las contingencias improvisadas que pueden aparecer en una obra en su práctica profesional.

A través del análisis del proceso de construcción, de los agentes participantes y del contenido documental del Proyecto Constructivo y sus especificaciones técnicas, se propone el aprendizaje en el manejo de las herramientas y documentación de la Dirección de Obra que la normativa dispone para su desarrollo (García Valcárcel, 2004). Así mismo, los procedimientos o rutinas para un correcto uso de las mismas frente a las contingencias que puedan aparecer durante una obra (Leceta Rey, 2017).

\section{Diseño del Ciclo de Mejora Docente}

\section{Mapa de contenidos y problemas clave}

Las 8 horas del ciclo de mejora que aquí se presenta están distribuidas en $4+4$ y se imparten los miércoles 9 y 16 de mayo de 2018. Los temas a desarrollar son:

- Tema 11: Supervisión de obra: Fachadas.

- Tema 12: Supervisión de obra: Revestimientos y carpinterías.

La estructura de las clases de supervisión previas al desarrollo de este ciclo son siempre similares: una parte 
teórica expositiva con presentación homogeneizada para todos los grupos de la asignatura y una parte práctica en la que el profesor revisa cómo van los trabajos de grupo a modo de tutoría particular.

La estructura de contenidos conceptuales de las clases de supervisión también es siempre similar para todos los temas y está dividida en tres bloques principales (García Valcárcel, 2004): normativa, definiciones/tipologías y procesos constructivos.

En la parte práctica, en base a la supervisión de sus prácticas de grupo que los alumnos realizan a lo largo del curso, se desarrollan los contenidos procedimentales destinados al Control y Análisis del documento del proyecto, la planificación de visitas, el control en obra y la resolución de contingencias.

Los contenidos actitudinales están presentes en toda la asignatura y se desarrollan más en las primeras clases en las que se hace hincapié en la responsabilidad civil y penal del arquitecto tanto en fase de proyecto como en fase de obra y en la importancia de la profesionalidad en la planificación, seguimiento y toma de decisiones en la fase de obra para conseguir un correcto resultado arquitectónico y constructivo.

A partir de los contenidos anteriores se ha realizado el mapa de contenidos (Figuras 1) en un esquema de nube con las actividades a desarrollar en ambas sesiones.

Jornadas de Formación e Innovación Docente del Profesorado | № 1 (2018) Esta obra se distribuye con la licencia Creative Commons 


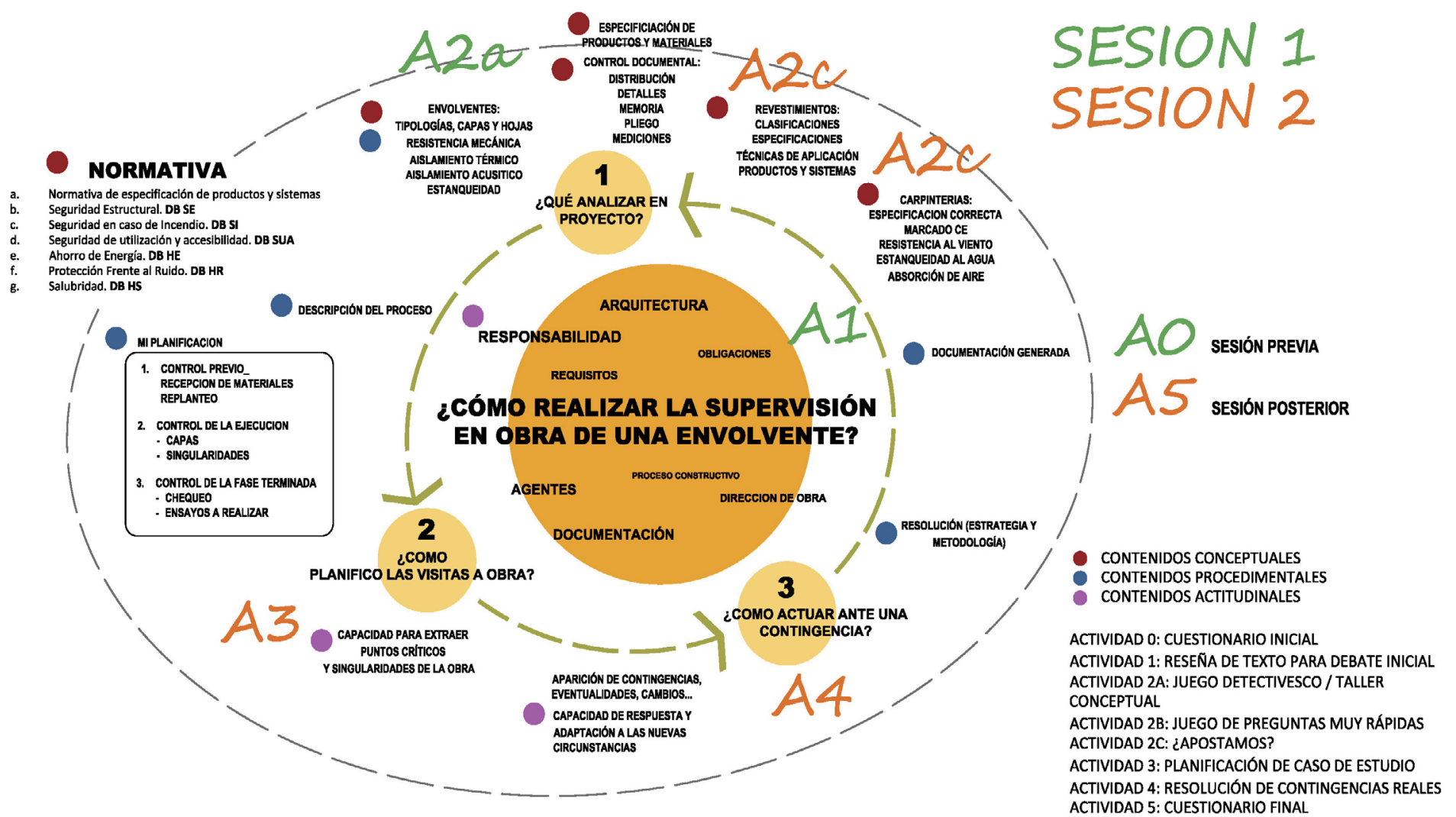

Figura 1. Mapa de contenidos de ambas sesiones de clase con las actividades correspondientes.

Jornadas de Formación e Innovación Docente del Profesorado | № 1 (2018)

(c) (i) $\ominus$ Esta obra se distribuye con la licencia Creative Commons

Internacional (CC BY-NC-ND 4.0.) 
Al tratarse del último curso, se ha pretendido diseñar un modelo basado en la reelaboración y reestructuración de las ideas que traen los estudiantes aprendidas (De Alba y Porlán, 2017).

Se ha estructurado el mapa en base a los contenidos procedimentales y actitudinales más que a los teóricos o conceptuales, ya que aquellos son fundamentales en esta asignatura.

Los contenidos conceptuales y las tres actividades dedicadas a éstos (A2a, A2b, A2c) son los que singularizan las sesiones. Dependiendo del tema, los alumnos se concentrarán en una parte específica de la obra. Se deben estudiar unas tipologías, productos y normativas concretas para cada caso. El resto de actividades serán comunes para los dos temas tratados.

El esquema gira alrededor de una problemática inicial relativa al tema en cuestión y se subdivide en tres fundamentales que corresponden a las tres fases de la dirección de obra y que podríamos considerar los contenidos procedimentales básicos:

1. ¿Qué analizar del proyecto?

2. ¿Cómo planificar las visitas?

3. ¿Cómo actuar ante una contingencia?

Es un bucle cerrado en círculo porque realmente la resolución de una contingencia en obra debe concluir con unas modificaciones del propio proyecto, que se transforma como un documento vivo, para que éste al final refleje lo ejecutado realmente en obra enmarcado siempre en la Normativa de aplicación y que corresponde a la responsabilidad última del Director de Obra (Peñin, 2006).

\section{Modelo metodológico y secuencia de actividades}

El modelo metodológico ha estado basado en la resolución de problemas y sobre todo en técnicas próximas a la línea de "dar clase con la boca cerrada" (Finkel, 2008). Todo ello apoyado en la incorporación de la gamificación en casi todas las actividades programadas. 
Una de las singularidades de la programación es la gestión de la intensidad de las sesiones. Al ser sesiones de cuatro horas, esta cuestión se presenta como fundamental para poder establecer transiciones y secuencias entre actividades dinámicas con intensidad alta y otras más moderadas y reflexivas.

Se ha tratado de abarcar contenidos procedimentales equivalentes de ambos temas con una misma actividad y así no repetir actividades en ambas sesiones. De esta manera se ha podido experimentar con ritmos e intensidades muy diferentes. En la primera sesión se desarrolla un "taller conceptual" (Finkel, 2008) de tres horas y en la segunda una secuencia más dinámica con cuatro actividades de 60 minutos. En el mapa se han señalado las actividades por dos colores: verde las de la primera sesión y naranja para la segunda sesión.

Tabla 1. Esquema con la secuencia de actividades programadas.

\begin{tabular}{|l|l|l|l|}
\hline SESION 1 & & & \\
\hline ACTIVIDAD 1 & $9-05-2018(9: 00 \mathrm{~h})$ & $\begin{array}{l}30 \\
\mathrm{~min} .\end{array}$ & LECTURA Y DEBATE \\
\hline ACTIVIDAD 2a & $9-05-2018(9: 30 \mathrm{~h})$ & $\begin{array}{l}210 \\
\mathrm{~min} .\end{array}$ & $\begin{array}{l}\text { JUEGO } \\
\text { DETECTIVESCO }\end{array}$ \\
\hline SESION 2 & & & \\
\hline ACTIVIDAD 2b & $16-05-2018(9: 00 \mathrm{~h})$ & $\begin{array}{l}60 \\
\mathrm{~min} .\end{array}$ & $\begin{array}{l}\text { CONCURSO DE } \\
\text { PREGUNTAS }\end{array}$ \\
\hline ACTIVIDAD 2c & $16-05-2018(9: 30 \mathrm{~h})$ & $\begin{array}{l}60 \\
\mathrm{~min} .\end{array}$ & ¿APOSTAMOS? \\
\hline ACTIVIDAD 3 & $\begin{array}{l}16-05-2018 \\
(12: 00 \mathrm{~h})\end{array}$ & $\begin{array}{l}60 \\
\mathrm{~min} .\end{array}$ & $\begin{array}{l}\text { PLANIFICACION DE } \\
\text { CASO DE ESTUDIO }\end{array}$ \\
\hline ACTIVIDAD 4 & $\begin{array}{l}16-05-2018 \\
(12: 00 \mathrm{~h})\end{array}$ & $\begin{array}{l}60 \\
\mathrm{~min} .\end{array}$ & $\begin{array}{l}\text { ANALISIS DE CASOS } \\
\text { REALES }\end{array}$ \\
\hline
\end{tabular}

El sentido de la secuencia de actividades que se representa en el mapa (Figura 1) y en la Tabla 1 es el que se expone a continuación. 
Las actividades numeradas en la figuras 1 como A0 y A5 corresponden a la realización en clase de los cuestionarios inicial y final y se realizan antes y después de las dos sesiones para poder comparar la evolución de los conocimientos trabajados.

En la actividad 1 (Tabla 1) se plantea la lectura en grupo del texto: "La transformación del proyecto arquitectónico durante el proceso constructivo" (Peñín, 2006), para posteriormente realizar un debate sobre el texto a modo de inicio o precalentamiento de la clase.

El contenido desarrollado es básicamente actitudinal y en él podríamos destacar el posicionamiento que debemos tener ante la labor profesional de la dirección de obras sin perder la finalidad última de la misma, que es generar un hecho arquitectónico. Para ello tenemos que ser conscientes de que debemos realizar un trabajo basado en la disciplina, el conocimiento del proyecto y con una actitud diligente con el cumplimiento de la normativa y la labor social del arquitecto.

La actividad A2a es una "taller conceptual" de tres horas en las que se pretende que el alumno tome conciencia de que vamos a aprender de otra manera, de la importancia de la investigación y la implicación personal en el aprendizaje y cómo esto se puede aplicar al desarrollo de su profesión (Finkel y Barberá, 2008). La implicación personal permite un mejor posicionamiento ante cualquier situación y una mejor capacidad para dar una respuesta crítica ante cualquier contingencia (Gargallo, Suárez y Ferreras, 2007).

Los contenidos desarrollados son los conceptuales correspondientes al tema de Fachadas, además de contenidos actitudinales genéricos: ser responsable con nuestras obligaciones y nuestras decisiones de proyecto, hasta el punto de dejar la dirección de obras por ver en peligro nuestra responsabilidad.

Se trata de sustituir una clase expositiva de los contenidos teóricos por una actividad más participativa y sobre 
todo que genere la implicación de los alumnos. Se propone realizar un "juego detectivesco" en el que tienen que resolver un caso a partir de una premisa inicial y unas pistas que se facilitan de forma paulatina o que tienen que conseguir en internet. La labor del profesor es la de guiar el desarrollo de su investigación.

La actividad A2b tiene el formato de concurso de preguntas y respuestas muy rápidas, de una complejidad muy concreta y homogénea. Básicamente lo que busco es que aprehendan agilidad para identificar productos, técnicas y denominaciones normativas para los diferentes tipos, que son muchos, de revestimientos que aparecen en el temario.

La dinámica será muy rápida, aunque exigente al tiempo que divertida. Las preguntas están divididas en seis colores, por temáticas y deben conseguir tres de cada color para completar el juego. Se acercan a la mesa del profesor, tiran un dado para elegir color y se les asigna por mi parte una tarjeta con una pregunta. Vuelven a su mesa, la responden en común y vuelven corriendo al profesor para entregar la respuesta. Si es correcta repiten la operación y si no lo es tienen que volver a su mesa a generar una nueva respuesta.

La actividad A2C es específica de los contenidos conceptuales del tema de carpinterías. Es una actividad con un sentido mucho más concreto. El objetivo es el manejo de determinadas nomenclaturas y especificaciones a las que los alumnos son ajenos. Las actividades $2 \mathrm{a}, 2 \mathrm{~b}$ y $2 \mathrm{c}$ son mucho más dinámicas y exigen un grado de implicación mayor. Se trata de un ejercicio práctico en el que les pido a los estudiantes especificar completa y correctamente la carpintería del aula ayudados por los apuntes y bibliografía (Orozco Sánchez, 2014)"author":[\{“dropping-particle":"',"family":"Orozco Sánchez","given":"Teresa","non-dropping-particle":",",parse-names":false,"suffix":"”\}],"id":"ITEM-1","issued":\{“date-parts":[["2014"]]\},"publisher":"IC Editorial"," publisher-place":"Antequera (Málaga. 
La actividad 3 está encaminada a desarrollar contenidos procedimentales comunes a ambos temas. Es este caso el de la planificación de las visitas a obra. La planificación debe contener los aspectos que irían a controlar a obra de esa fase en concreto si pudiesen acceder a ella sin limite, cosa que en realidad no pueden hacer.

El control deben estructurarlo según el Código Técnico de la Edificación (Ministerio de la Vivienda, 2007) en:

C0. à 7.2. Control previo: recepción en obra de productos, equipos y sistemas.

C1. à 7.3. Control de ejecución de la obra.

C2. à 7.4. Control de la obra terminada.

La actividad 4 también tiene como finalidad el desarrollo de objetivos procedimentales comunes y se trata de la resolución de contingencias de obra. Esta última actividad es más conclusiva porque en ella deben aplicar todo lo aprendido hasta ahora en el caso práctico que están desarrollando. También a nivel de intensidad de trabajo es bastante menor, con vistas a finalizar la sesión, pues se trata de revisar lo que están haciendo en sus obras y resolver dudas.

\section{Aplicación del Ciclo de Mejora Docente}

\section{Relato resumido del desarrollo de las actividades}

A continuación se exponen los resultados de la aplicación de las actividades en ambas sesiones de clases y los ajustes y comentarios que han surgido durante el proceso.

Actividad 1: Lectura y debate

Este debate inicial era sólo para precalentar la clase. Se debate el posicionamiento de la asignatura dentro de la estructura general de la carrera. La dirección de obra es una parte de la generación de la arquitectura. Una máxima personal que suelo aportar en estos debates es: "Una mala 
dirección de obras puede estropear un magnifico proyecto y dar como resultado una mala arquitectura, pero en cambio, una buena y profesional dirección de obra puede convertir un mal proyecto en una arquitectura digna".

Actividad 2a: Juego detectivesco / Taller conceptual

Se utilizaron los 210 minutos, gracias a la secuencia de las pistas que se iban aportando por parte del profesor a lo largo de la clase.

Esta actividad requiere mucho trabajo previo pero es tremendamente enriquecedora en cuanto a los contenidos desarrollados, y sobre todo en cuanto al desarrollo del sentido crítico del estudiante. A medida que avanzaban las pistas, sus preguntas eran cada vez más acertadas, concisas y sobre todo profundas. Pienso que es una actividad muy adecuada para desarrollar diferentes niveles de profundidad en los contenidos (Rivero y Porlán, 2017).

Actividad 2b: Concurso de preguntas muy rápidas

La programación de 60 minutos se quedó corta. Pero se terminó el juego a petición de los propios estudiantes, y se tuvo que ajustar, por ello, el tiempo de las actividades posteriores.

Su enfoque, como ya he comentado, no es el de fomentar el sentido crítico ni la profundidad en los conceptos. Es más un estilo ágil para que aprendan a familiarizarse con cuestiones que desconocen totalmente. En este caso nomenclatura y especificaciones muy diversas. Fue divertido para mi y para ellos con la escenografía de las tarjetas de colores y el hecho de tener que ir y volver a la mesa del profesor a elegir color. Es importante que ellos mismos, durante el transcurso de la actividad, vayan tomando conciencia de las lagunas que tienen en determinados campos.

Actividad 2c: ¿Qué apostamos?

La programación de 60 se ajustó a 45 minutos debido al desarrollo de la actividad anterior. Se ajusta a tiempo de manera sencilla aprovechando que de los once puntos solicitados inicialmente en la actividad solamente siete son 
aspectos obligatorios por normativa para una carpintería. Por lo tanto se reduce el contenido de la actividad a estos siete aspectos fundamentales.

Actividad 3: Planificación de obra

La programación de 60 tuve que pasarla a 45 minutos debido a la actividad anterior.

Poco a poco se va bajando el ritmo de la clase. Es una actividad nada competitiva y ya se trabaja por inercia. Bastante aceptable contando con que venimos de la efusividad de las actividades anteriores. Es una actividad tranquila e interesante para coger un automatismo de planificación, contenido procedimental necesario que los alumnos deben incorporar en su quehacer diario profesional.

Actividad 4: Análisis de casos reales

Es interesante la exposición de sus trabajos ya que existe una gran diversidad en cuanto a tipologías de edificios a visitar, así como de la normativa de aplicación en cada caso; también interesa porque hay disparidad en cuanto a las fases de obra analizadas. De esta manera pueden ver muchas más fases entre todos. Lo interesante de esta actividad es que los estudiantes vean que, con independencia de cada obra, siempre debemos tener una metodología para enfrentar cada contingencia. Siempre hay un patrón común al que podemos acudir como modelo de respuesta.

\section{Cuestionario inicial y final}

Los dos cuestionarios han sido idénticos. Se trata de responder de forma reflexiva a cuatro preguntas relacionadas con cuatro campos conceptuales. La finalidad básica ha sido utilizar los resultados para diseñar la secuencia de actividades de las sesiones (Rivero y Porlán, 2017).

La pregunta 1 pretende relacionar conceptos aprendidos en asignaturas de cursos anteriores con una estructura sistemática para especificar unidades que aplicamos en este curso. Se les pide que identifiquen y especifiquen 
sobre un detalle constructivo mudo una serie de productos numerados.

La pregunta 2 pretende desarrollar un sentido crítico a la hora de seleccionar aspectos importantes en la planificación de obra y relacionarlo con cuestiones que influyen en las responsabilidades de la dirección de obra.

La pregunta 3 está encaminada a conocer el dominio de la normativa que deben utilizar para estos sistemas constructivos en concreto y si usan la terminología concreta.

La pregunta 4 es una pregunta en la que se pretende comprender el grado de madurez del estudiante a la hora relacionar obligaciones profesionales concretas con la implicación a nivel de responsabilidad para/con la sociedad que conlleva de la función del arquitecto.

Los cuatro gráficos de la Figura 2 recogen sintéticamente los resultados del pase de los cuestionarios, siguiendo el modelo de "escalera de aprendizaje" (Rivero y Porlán, 2017).

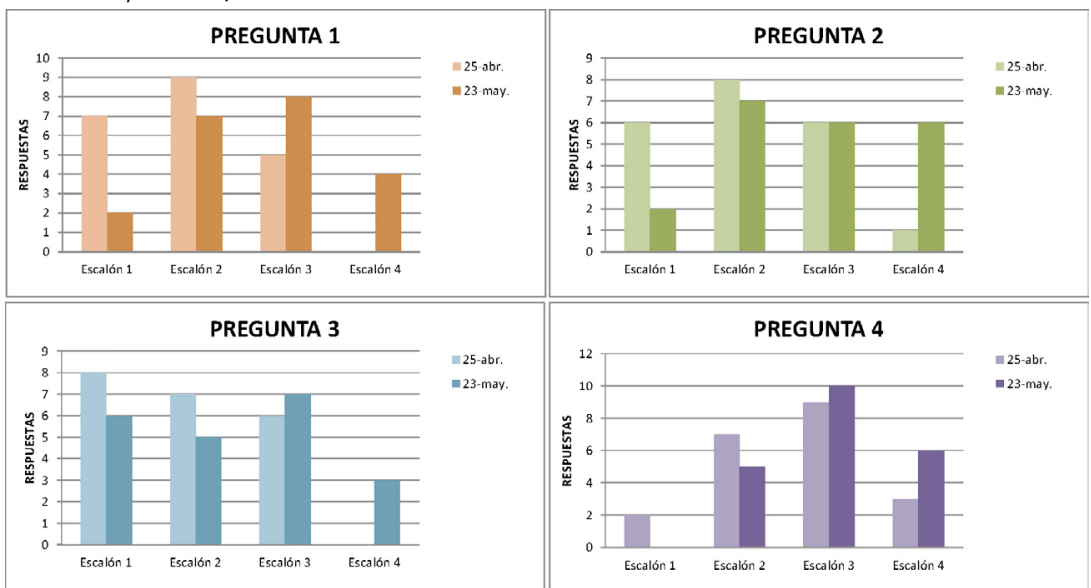

Figura 2. Respuestas de las cuatro preguntas del cuestionario organizadas en escalera.

Los escalones corresponden al grado de complejidad y manejo de conceptos manifestado por el estudiante en cada respuesta y podrían resumirse así: 
Escalón 1: Tiene un criterio disperso y poco concreto sobre los aspectos a manejar.

Escalón 2: Establece relaciones entre los contenidos expuestos con argumentos excesivamente sencillos o inocentes.

Escalón 3: Establece relaciones adecuadas y argumenta con palabras y términos no del todo adecuados técnicamente.

Escalón 4: Establece relaciones y argumenta correctamente utilizando terminología y conceptos adecuados.

\section{Análisis de resultados del cuestionario inicial}

La interpretación de los resultados de forma escalonada permite, sobre todo, tener una orientación del estado de la clase que me ha servido de mucho, ya que son estudiantes de último curso y es muy interesante saber cómo llegan a este curso con la amalgama de conocimientos, en general, aprendidos en años anteriores. Me ha sorprendido gratamente el grado de madurez general apreciable en la pregunta 2 y sobre todo en la 4 . En las preguntas 1 y 3 hay falta de coherencia en el manejo de conceptos y sobre todo de terminología, lo que me hizo planificar para la sesión 2 la actividad concreta (A2b) para el manejo de terminología en cuanto a revestimientos. Las especificaciones técnicas requieren una madurez en la utilización de una gran cantidad de conceptos aprendidos en cursos y asignaturas diversas que normalmente los alumnos no suelen alcanzar ni siquiera cuando terminan la carrera.

\section{Análisis de resultados del cuestionario final}

Tengo claro que este cuestionario no me podría servir para calificar porque mi forma de evaluarlo ha estado fundamentada más en la madurez y capacidad para argumentar y crear un discurso coherente que en la exactitud de 
los conceptos expuestos. No obstante, se ha sido bastante exigente con el último escalón en el que sí se valoran esos aspectos. En general se han producido avances relevantes en todos los resultados y algunos han sido notorios.

Tal y como se ha comentado antes, se hace hincapié en reforzar la estructura con la que deben especificar los productos, ya que los conceptos los tienen y con una buena estructura que les ayude y les exija organizar sus ideas serán capaces de realizar un buen trabajo.

Con respecto a la planificación han alcanzado buenos resultados, debido a que es un tema que prácticamente se aborda a diario, en la parte teórica o en las prácticas. Por el contrario, el bagaje que traen en cuanto a manejo de normativa no puede ser mejorado en la asignatura, pues es una materia a la que no podemos dedicarle más que un repaso en cada tema.

De nuevo, la pregunta 4 es la más sorprendente, no por la evolución, ya que parten de unos buenos resultados desde el cuestionario inicial, sino porque se aprecia un buen nivel en cuanto a argumentación y conciencia de la responsabilidad profesional.

\section{Conclusiones finales}

Sintetizo a continuación algunas conclusiones básicas de la experiencia innovadora desarrollada.

Sobre el aprendizaje y la relación con los estudiantes

Tener como principio que los protagonistas de las sesiones sean los estudiantes ha generado una metamorfosis que sentía como necesaria desde que se implantó el Plan Bolonia. Este cambio de plan conllevó en su momento una reducción de horas y una concentración de la materia.

Con este ciclo hemos conseguido generar actividades interesantes para ellos y ello se ha reflejado en una actitud muy participativa e incluso en una maduración, según 
pasaban las clases, en el uso del lenguaje técnico y de los argumentos usados por su parte.

En este aspecto del diseño, me ha resultado de lo más útil haber realizado el cuestionario inicial para poder diseñar la metodología adaptada a las necesidades de la clase. También ha sido importante realizar actividades más básicas como el juego de preguntas cortas (Act. 2b) para mejorar determinados aspectos de terminología o actividades en las que se genere más reflexión y debate sobre contenidos actitudinales como el taller conceptual (Act. 2a).

Sobre los contenidos de enseñanza

Del ciclo hay dos cuestiones fundamentales en las que he basado el tratamiento de los contenidos de las dos sesiones.

En primer lugar he tratado de jerarquizar los contenidos. Darle prioridad a determinadas cuestiones que giran alrededor de una problemática inicial relativa al tema a tratar. Es un ejercicio fundamental, pues en esta asignatura de último curso se barajan contenidos de todos los cursos anteriores y es necesario priorizar lo que es realmente esencial de esta asignatura.

En segundo lugar creo que lo más relevante ha sido organizar gráficamente el mapa de contenidos en base a contenidos procedimentales y actitudinales, dejando en segundo orden los conceptuales o teóricos. Esto me ha ayudado a tenerlos presentes en el diseño de las actividades.

Sobre el modelo metodológico y los principios didácticos

Fue muy útil la presentación en la clase del Curso General de Docencia Universitaria, ante mis compañeros, de la primera sesión planificada, tras la cual me hicieron ver entre todos que esas primeras cuatro horas estaban muy saturadas y que no tendría tiempo material para llevarlas a cabo. Tenía programado el taller conceptual y dos actividades más, quizás llevado por mi inexperiencia y por no creerme que podría tener a la clase tres horas y media realizando un taller conceptual (juego detectivesco). Esto 
sucedió asi y sobre la marcha entre la primera y la segunda sesión adapté las actividades y realicé un diseño de la segunda sesión acomodando actividades que tenía programadas en la primera.

Por último, resaltar lo interesante de la incorporación de modelos de actividades diferentes. La primera sesión fundamentada en un taller conceptual ha permitido profundizar en contenidos y plantear debates y reflexiones profundos y concisos. En la segunda sesión, en efecto, había una necesidad por mi parte de resolver una falta de conocimientos básicos, y a través de un juego (con unas reglas sencillas, pero que me permiten implicar a los estudiantes en una dinámica de búsqueda) se fue dirigiendo la clase a una situación de participación que me hizo incluso olvidarme de la finalidad última de la actividad. Aunque era un juego, es revelador que fueron los propios alumnos los que me exigieron llegar al final para obtener un resultado concreto generado por la competición.

Modificar de esta manera la forma de trabajar los contenidos ha sido de lo más sorprendente. Según íbamos realizando las actividades en clase no daba crédito a la participación de los estudiantes y a la dinámica en la que entraba una clase que normalmente era distante y con una relación de pasividad absoluta, en la que yo hablaba y ellos escuchaban.

Jornadas de Formación e Innovación Docente del Profesorado | № 1 (2018) Esta obra se distribuye con la licencia Creative Commons 


\section{Referencias bibliográficas}

De Alba, N. y Porlán, R. (2017). La metodología de la enseñanza. En R. Porlán (Coord.), Enseñanza Universitaria: Cómo mejorarla (pp. 37-54). Madrid: Morata.

Finkel, D. (2008). Dar clase con la boca cerrada. Valencia: Universitat de València.

García Valcárcel, A. (2004). Manual de dirección y organización de obras. Madrid: C.I.E. Dossat 2000.

Gargallo, B., Suárez, J. y Ferreras, A. (2007). Estrategias de aprendizaje y rendimiento académico en estudiantes universitarios. Revista de Investigación Educativa, 25, 421-441.

Leceta Rey, A. (2017). Manual de dirección y control de obra. Guadalajara: Colegio Oficial de Aparejadores, Arquitectos Técnicos de Ingenieros de Edificación de Guadalajara.

Ministerio de la Vivienda (2007). Código Técnico de la Edificación. Recuperado de https://www.codigotecnico.org/

Orozco Sánchez, T. (2014). Experto en aplicacion del CTE a la dirección de obra: Documentación de control. Málaga: IC Editorial.

Peñín, A. (2006). La transformación del proyecto arquitectónico durante el proceso constructivo. La Ópera de Sídney y el Centro Pompidou de París. Barcelona: UPC.

Rivero, A. y Porlán, R. (2017). La evaluación en la enseñanza universitaria. En R. Porlán (Coord.), Enseñanza Universitaria: Cómo mejorarla. (pp. 73-92). Madrid: Morata.

Jornadas de Formación e Innovación Docente del Profesorado | № 1 (2018) Esta obra se distribuye con la licencia Creative Commons 
